\title{
Mechanochemical Sorption Apparatuses
}

\author{
Chuntonov Konstantin ${ }^{1, a}$, Lee Man Kyu ${ }^{2, b}$ \\ ${ }^{1}$ Yael 30, Nesher, 36760, Israel \\ ${ }^{2}$ C-1816, PoongLim I-want Plus, 255-1, Seohyeon-Dong, Bundang-Gu, Sungnam-City, \\ Kyoungki-Do, 463-862, Korea \\ a konstantin@chuntonov.com, ${ }^{\mathrm{b}} \mathrm{mklee}$ @@hanafos.com
}

Keywords: high purity gases, mechanochemistry, control of the sorption process.

\begin{abstract}
A new method of sorbent activation by its mechanical grinding directly in the purified gas medium promises a technological breakthrough in the field of manufacturing and using of high purity gases. Simple design solutions and using reactive alloys as a sorbent allow making the gas purification process controllable and reducing the cost of this process by many times as well as reducing the concentration of the impurity in the end product.
\end{abstract}

\section{Introduction}

The efficiency of the sorption process essentially depends on the value of the sorbing surface and hence on the dispersity of the sorption material. The higher the dispersity of the sorbent the faster and more complete the process.

In the current technologies dispersion of the solid sorbent and the sorption process are separated in time and space: first the sorbent is produced in one place and then it is transferred into another place, where the sorption of gases or liquid components takes place. This scheme is long established; however, besides a number of advantages it has its drawbacks.

The described below mecanochemical sorption apparatuses do not have the mentioned drawbacks in principle because opposite to the old technologies the new one is based on the combination of these two production stages, dispersion and its reaction with the sorbed substance, so both of them take place in the same volume and in the same time. The mentioned combination is achieved with the help of simple design solutions when reactive ingots are mechanically grinded into powder directly in the treated liquid or in the gas flow.

\section{The Technical Description of the Mechanochemical Method}

Sorption apparatuses with mechanochemical activation of sorption material are applicable to practically all gases and liquids in any of their states. However, here we will limit ourselves to the discussion of only application of the new technology - controlled purification of flow gases. This example is sufficient both for the general understanding of the essence of the mechanical method and for the illustration of its possibilities.

In the instrumental respect the given technology is rather simple. Milling of the initial ingot with a monolithic structure is performed inside the sorption column mechanically in the disintegrator 2 (Fig.1) without a sealing failure in the column. 


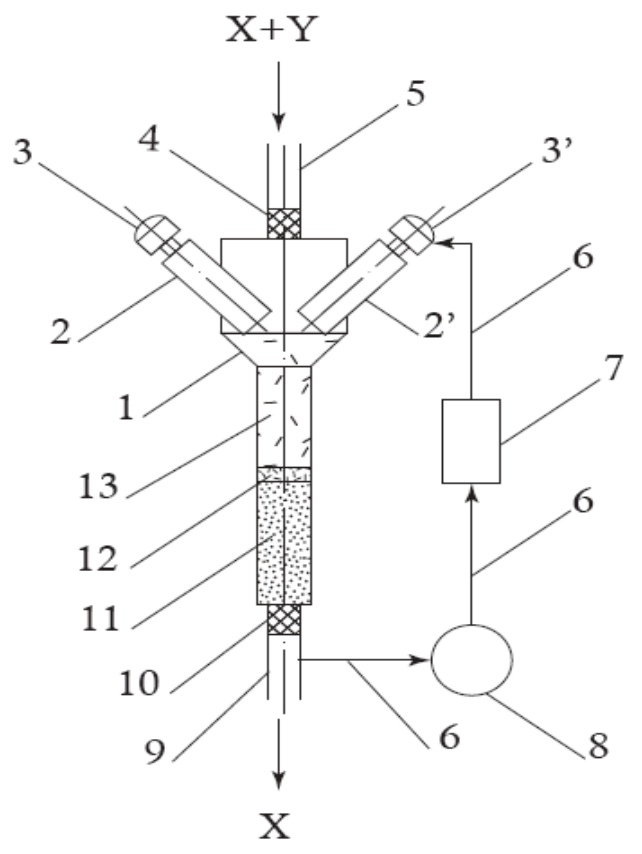

Fig. 1. The scheme of the sorption apparatus (K. Chuntonov, Sorption apparatus for the production of pure gases, Patent Application WO 2012081004, June 21, 2012)

1 - the impermeable to gas casing of the sorption column, 2 and 2' - disintegrators, 3 and 3' - outside actuators, $4-\mathrm{a}$ filter, 5 - an inlet, 6 - a feedback line, 7 - a controller, 8 - a gas analyzer, 9 - an outlet, 10 - a filter, 11 - used powder, 12 - a layer of fresh powder, 13 - falling particles.

The products of milling in a form of particles 13 pour out of the disintegrator forming a powder column, the upper part of which consists of fresh powder 12 and the lower - of the exhausted particles 11. Gas X with impurity $\mathrm{Y}$ is fed from above through filter 4 passes through the column of reactive particles and exits in the purifies form through the lower filter 10.

The time law of cutting and milling the surface layers of the ingot in the disintegrator can either follow a strictly set program or can be adjusted for the current requirements of the process. In the latter case analytes of the exiting gas $\mathrm{X}$ are taken and analyzed by device 8 , which transmits the information further to controller 7, which regulates the intensity of the work of the actuator 3. This actuator is connects via a feedthrough to the milling tool, which is located inside the disintegrator. So by changing the intensity of milling it is possible to adjust the sorption rate to any gas flow rate obtaining at this the end product with the set purity level.

Comparing the mechanochemical method with the existing technologies of manufacturing pure and high purity gases one can see many advantages of the new method. Adsorption methods, which are using molecular sieves, require cooling the material to cryogenic temperatures; at this the material itself is too much selective. The methods of getter purification, on the opposite, require heating of the sorption material. Both lead to the complication of the design of the sorption apparatuses and make their operation more expensive.

The new method compares favorably to the traditional technologies, it is economical, universal and extremely effective. Here are its main advantages.

Super Reactivity of As - Milled Powders. Powders produced by mechanical disintegration of solid material in the cold state are supersaturated with defects of different origin and for this reason are super reactive. The abrupt decrease of activation barriers is characteristic for them both for reactions and for the diffusion processes. Temperature thresholds of the sorption processes decrease and the processes themselves become faster. 
Controllability of the Sorption Process. Mechanochemical apparatuses allow controlling the sorption process by varying the amount of the powder mass produces in a time unit. It is possible select such a grinding rate for each flow rate, that a stabilized regime will be set, when the amount of the impurity Y (Fig. 1) entering the sorption column is equal to the amount of impurity captures by metallic particles Me in a form of a chemical compound MeY. In such regime the exiting gas has constant purity.

Extremely High Efficiency. The new technology is applicable to any sorption materials; however, ingots of reactive metals are in the given case preferable. The mentioned alloys, which consist mainly of alkali or alkali-earth metals, continuously sorb gases at room temperature following either the linear or the parabolic law i.e. the reaction with gases runs to completion. This is an important point allowing limiting the grinding to the powder size of a micron scale with the particle size from some microns to some hundreds of microns, which is a routine procedure done with the help of standard tools. At this the ingots are grown with high rate in metallic ampoules according to the classical Stockbarger's method.

Simplicity and Safety. Mechanochemical sorption columns have simple design and convenient in servicing, do not require heating or cooling and at the same time have much smaller dimensions than the known apparatuses of the same capacity. The new technology is safe both for the personnel and from the point of view of the preservation of the purification material. In the case of unauthorized entrance of the air inside the apparatus the ingot surface is immediately covered by a thin layer of compounds, which abruptly slow down the rate of the reaction, and the losses of the powder mass come down only to a thin upper layer of fresh particles.

\section{Economic Analysis}

Economic analysis of the situation in the field of pure gases leads to interesting and far going conclusions; in particular, to the conclusion that the traditional ways of gas purifying and the mechanochemical method do not compete but mutually add to each other forming together one multistage technology, which revolutionizes the industry of pure and super pure gases.

Price indices of the known purification methods and those of the new method are compared in Fig.2, where the averaged data on $\mathrm{Ar}$ and $\mathrm{H}_{2}$ related to $50 \mathrm{~L}$ of gas under the pressure $200 \mathrm{bar}$ are given as examples. Gas of $99.9 \%(3 \mathrm{~N})$ of purity is taken here for the initial state.

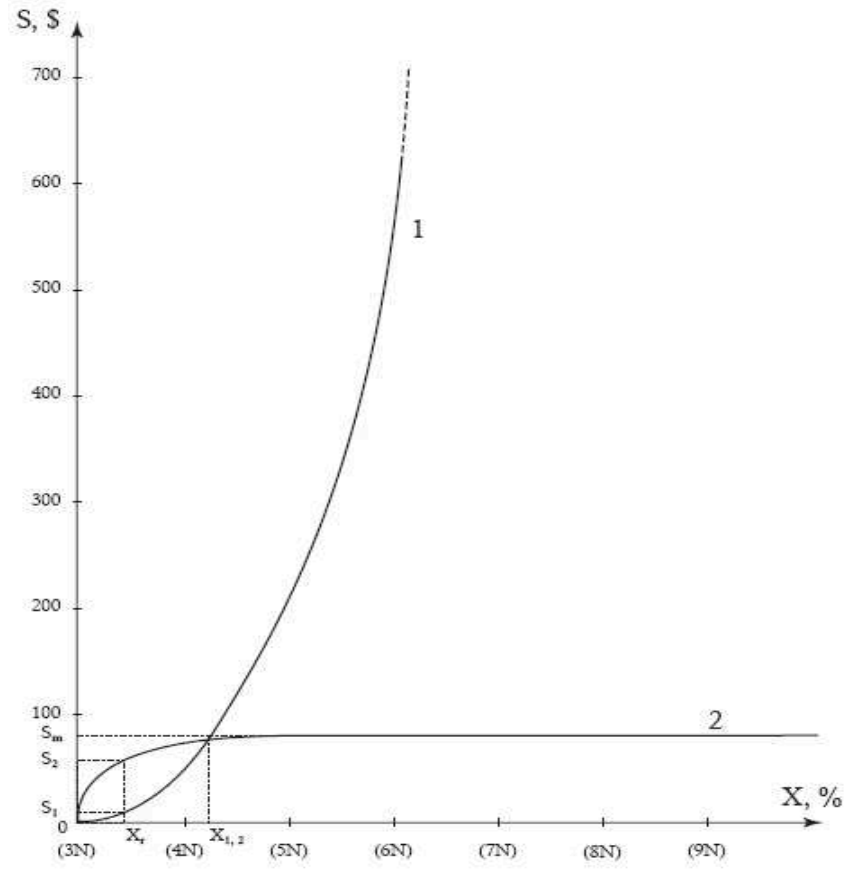

Fig. 2. Dependence of the price of the gas product on its purity level: 
1 - traditional purification methods, 2 - purification in mechanochemical apparatuses; $x$ concentration of the main substance in the gas product, $x_{\mathrm{r}}-$ the optimal concentration for the transition from the traditional treatment to the mechanochemical purification, $x_{1,2}-$ gas concentration, where the prices for the compared methods are equal, $s-$ the price of $10 \mathrm{~m}^{3}$ of gas at normal temperature and the pressure of $1 \mathrm{bar}, s_{\mathrm{m}}$ - the price of extremely pure gas according to the mechanochemical technology, $s_{2}$ - the price of gas with the purity $x_{\mathrm{r}}$ in the mechanochemical purification, $s_{1}-$ the price of gas with the purity $x_{\mathrm{r}}$ in the traditional technology.

The most sufficient peculiarity of the curves price / purity (Fig.2), which characterizes the old 1 and the new 2 technologies is that both dependences have a form of limit curves but with the different signs of curvature: while in the current gas purification methods the expensed grow progressively with each next purity level (curve 1), in the case of the mechanochemical purification the expenses rapidly decrease till they become negligibly small (curve 2 ).

This is clear, because during the long time of their operation the traditional methods hit the ceiling of their possibilities whereas the mechanochemical method in its very nature is the most effective method of manufacturing high purity gases. As-made powders react with gas impurity instantaneously and the amount of the consumed metal in chemical respect is equivalent to the amount of the sorbed impurity. So the higher the purity of the treated gas the smaller is the consumption of the metal.

It follows from Fig. 2 that whatever the demand for gases of the purity $7 \mathrm{~N}$ and higher, the price for this kind of product if the old technologies are used (even if they manage to overcome a number of technical problems arising at this) will be as seen from the curve 1 unacceptably high, being more expensive than the gas of the purity $6 \mathrm{~N}$ as a minimum by an order of magnitude.

It also follows from Fig. 2 that with the appearance of the mechanochemical purification the usage of the traditional technologies becomes economically unprofitable in the production of gases with the purity higher than $x_{1,2}$, where $x_{1,2}$ is the coordinate of purity defined by projection of the intersection point of the curves 1 and 2 onto the axis $x$.

This leads to the idea of a combined method, in which after achieving the level $x_{1,2}$ the traditional gas treatment is replaced by mechanochemical purification, which will allow obtaining super pure gases for the fixed price $s_{\mathrm{m}}$. The given price corresponds to the consumption of reactive metal necessary for sorbing $0.1 \%$ of impurities in $10 \mathrm{~m}^{3}$ of gas under standard conditions.

It finally follows from Fig. 2 that even such moderate price as $s_{\mathrm{m}}$ (approximately from 50 to $80 \$$ depending on the sort of the purified gas) can be considerably decreased if the transition to mechanochemical purification is carried out in a certain neighborhood of the point $x_{\mathrm{r}}$, which belongs to the vertical connecting the farthest points of the curves 1 and 2 . In this case the total price $s_{\mathrm{r}}$ of the gas produced according to the combined method is formed as a sum $s_{\mathrm{r}}=s_{1}+\left(s_{\mathrm{m}}-s_{2}\right)=s_{\mathrm{m}}-\left(s_{2}-s_{1}\right)$, which is much less than $s_{\mathrm{m}}$. Using catalogue prices for pure reactive metals we come to the price interval of $10 \$ \leq s_{\mathrm{r}} \leq 25 \$$, which will now determine the cost of gases of the highest purity.

It is difficult to imagine all the consequences of implementation of mechanochemistry into gas purification practice. However, there is no doubt that all the users of pure gases will get a strong impulse for the developments in their fields buying gases of the purity of $8 \mathrm{~N}, 9 \mathrm{~N}, \ldots 12 \mathrm{~N}$, etc. for the price of about $20 \$$ for a $50 \mathrm{~L}$ cylinder at the pressure of 200 bar.

\section{Conclusion}

Mechanochemical purification, i.e. purification by grinding the solid sorbent directly in the purified media during the sorption process is a new method of the final stage of purification of liquid or gaseous substances. The given method is applicable both at high pressures the sorption apparatus and at low pressures and also both in the range of room temperatures and in the range of cryogenic temperatures till $-200^{\circ} \mathrm{C}$ when chemical reactions between alkali or alkali-earth metals and the treated liquid are still observed. 
Sorption apparatuses with mechanical activation of reactive ingots are intended for the usage in the following processes and for the following purposes (with the proper selection of the sorption material): for deep purification of flow gases; for additional purification of nonaqueous solutions as well as liquid reagents of organic nature; for purification of liquefied gases in Dewar's vessels; for purification of rarefied gases or for vacuum maintenance in the chambers supplies with the means of primary pumping down like scroll pumps, diaphragm pumps, etc.

Besides, apparatuses with a transparent wall can become a new instrument for the research or for the demonstration of mechanochemical effects due to their visualization. Apparatuses of this kind will facilitate the appearance and spreading of the new knowledge in the field of mechanochemistry, which for sure is enjoying a boom at the present time.

The estimations based on the data about gas permeability of stainless steels and on the data on partial pressure of the products $\mathrm{MeY}$ close to room temperature show that mechanochemical purification of substances is able to decrease the content of active and low activity impurities in them to $\sim 10^{-15} \%$.

\section{References}

[1] K. Chuntonov, Patent Application WO 2012081004 (2012).

[2] M. Senna, in: Mechanochemistry, edited by H. Masuda, K. Higashitani and H. Yoshida, Third Edition, Powder Technology Handbook, chapter, 2.14, CRC Press, Boca Raton (2006).

[3] S.L. James, C.J. Adams, C. Bolm, D. Braga, P. Collier, T. Friscic, F. Grepioni, K.D.M. Harris, G. Hyett, W. Jones, A. Krebs, J. Mack, L. Maini, A.G. Orpen, I.P. Parkin, W.C. Shearouse, J.W. Steed and D.C. Waddell: Chem. Soc. Review Vol. 41 (2012), p. 413.

[4] K. Meyer: Physikalisch - chemische Kristallographie, VEB Deutscher Verlag fur Grundstoffindustrie, Leipzig (1968).

[5] K.A. Chuntonov and S.P. Yatsenko: Recent Patents on Materials Science, Bentham Sci. Publishers, Vol.6 (2013).

[6] K. Chuntonov, Patent Application WO 2011145090 (2011). 